\title{
Radiação solar estimada com base na temperatura do ar para três regiões de Minas Gerais
}

\author{
Cláudio R. da Silva ${ }^{1}$, Valdiney J. da Silva ${ }^{2}$, \\ José Alves Júnior ${ }^{3} \&$ Hudson de P. Carvalho ${ }^{2}$
}

\begin{abstract}
RESU M O
A radiação solar incidente $\left(R_{s}\right)$ é uma variável importante em estudos agrícolas, particularmente para a estimativa da evapotranspiração e em modelos para produtividade. Entretanto, sua medição não é, em geral, realizada em estações meteorológicas convencionais. 0 objetivo deste trabalho foi avaliar nove modelos empíricos de estimativa de $\mathrm{R}_{\mathrm{s}^{\prime}}$ a partir da temperatura, para as regiões $M$ etropolitana, $V$ ale do Rio D oce e Zona da M ata em M inas Gerais. Dados de $\mathrm{R}$ diários foram obtidos por estações meteorológicas automáticas instaladas nessas regiões e pertencentes ao Instituto $\mathrm{N}$ acional de Meteorologia (IN MET). Para todos os modelos foram gerados coeficientes locais de calibração. 0 desempenho de cada método foi avaliado através dos seguintes indicadores: coeficiente de determinação $\left(R^{2}\right)$, raiz quadrada do quadrado médio do erro (RQME), erro médio (EM) e teste t. A pequena diferença entre os modelos avaliados indica que qualquer um desses modelos é passível de utilização mas, dado à simplicidade, desempenho e significância, o modelo de $\mathrm{H}$ argreaves, calibrado e com dois coeficientes, é o mais aplicável para estimar a radiação solar incidente.
\end{abstract}

Palavras-chave: calibração, equações empíricas, Hargreaves, Bristow-Campbell

\section{Estimation of solar radiation by air temperature models for three regions of M inas Gerais}

\begin{abstract}
A B STRACT
The incident solar radiation $\left(R_{s}\right)$ is an important variable in agricultural studies, particularly for the estimation of evapotranspiration and yield models. H owever, its measurement is not commonly performed in conventional meteorological stations. The aim of this study was to evaluate nine empirical models to estimate $R_{s}$ from the temperature for the $M$ etropolitan, Vale do Rio D oce and Zona da $M$ ata areas in Minas Gerais State, Brazil. The models used were Hargreaves, Annandale, Chen, Bristow \& Campbell, Donatelli $\&$ Campbell and $\mathrm{H}$ unt. Data used were obtained by $\mathrm{R}_{\mathrm{s}}$ daily automatic weather stations installed in these regions and belonging to Instituto $\mathrm{N}$ acional de Meteorologia (INMET). For all models local calibration coefficients were derived. The performance of each method was evaluated using the following statistical indicators: coefficient of determination $\left(R^{2}\right)$, root mean square error (RM SE), mean bias error (MBE) and test-t. The little difference between the models evaluated suggests that any of these models may be used. However, given the simplicity, performance and significance, the model of $\mathrm{H}$ argreaves, calibrated and with two coefficients, is the most suitable for estimating incident solar radiation.
\end{abstract}

Key words: calibration, empirical equations, Hargreaves, Bristow-Campbell

\footnotetext{
${ }^{1}$ ICIAG/U FU. Av. Amazonas, s/n, Bloco 2E, Campus U muarama, CEP 38900-402, U berlândia, M G. Fone: (34) 3218-2225, Ramal 204. E-mail:claudio@iciag.ufu.br

2 ICIAG/U FU. E-mail: neyjosilva@hotmail.com; hudsonpc@iciag.ufu.br;

${ }^{3}$ UFG. C.P. 131, CEP 74001-970, Goiânia, G0. E-mail: jose.junior@pesquisador.cnpq.br
} 


\section{INTRODUÇÃO}

A energia solar pode ser considerada a mais abundante fonte contínua de energia disponível para a espécie humana (AlSalaymeh, 2006). Por se tratar do principal fator meteorológico, a radiação solar diária incidente em uma superfície horizontal $\left(\mathrm{R}_{\mathrm{s}}\right)$ é de grande relevância em estudos ecológicos e ambientais, constituindo-se como principal fator condicionante da temperatura do ar, do solo, dos processos de evapotranspiração e dos modelos que simulam o crescimento e a produtividade de uma cultura (Trnka et al., 2007; Abraha \& Savage, 2008). A quantificação da radiação solar é necessária também para dimensionamento de sistemas geradores de energia solar fotovoltaica e energia térmica (Kaplanis \& Kaplani, 2007; Prieto et al., 2009; Almorox et al., 2011). Uma vez que a $\mathrm{R}_{\mathrm{s}}$ varia de acordo com a latitude do local, com as condições atmosféricas e posicionamento do Sol ao longo do dia, tornase imprescindível o monitoramento da sua variação espacial e temporal.

A radiação solar é medida por instrumentos como os radiômetros, actinógrafos bimetálicos e piranômetros, que também medem a radiação difusa, sendo este último o mais utilizado no Brasil (Dornelas et al., 2006). Entretanto, como em muitos outros países a medida direta da radiação solar ainda é escassa quando comparada com as medições de temperatura e precipitação. Esta situação reflete, provavelmente, não apenas o custo de aquisição do equipamento mas também os problemas de manutenção e calibração dos equipamentos que medem a radiação solar (Liu et al., 2009; Robaa, 2009). Mesmo nas estações que apresentam o equipamento para sua medição, existem dias nos quais os valores são perdidos ou apresentam inconsistência em função de falhas operacionais (Hunt et al., 1998).

Para localidades com ausência de coleta de dados de radiação solar, pode-se estimá-la através de relações matemáticas empíricas, interpolação linear, imagens de satélites geoestacionários e rede neural artificial, entre outros, conforme revisão feita por Liu et al. (2009). Esses métodos variam no grau de complexidade, dados de entrada e acurácia (Abraha \& Savage, 2008). Apesar disto, os métodos baseados nas relações empíricas são, em geral, mais atrativos devido à facilidade na aquisição dos dados. Os parâmetros comumente utilizados são: radiação incidente extraterrestre, insolação, temperatura do ar, nebulosidade, umidade relativa do ar, altitude, latitude e o número do dia do ano, podendo ser utilizados individualmente ou combinados entre si (Bristow \& Campbell, 1984; Menges et al., 2006; Li et al., 2010).

Uma relação empírica muito conhecida e com boa acurácia é o modelo cujo principal parâmetro de entrada é a insolação obtida por heliógrafo; entretanto, esse equipamento não está presente na maioria das estações agrometeorológicas. No intuito de resolver a limitação da disponibilidade de dados de insolação, Hargreaves (1981) propôs um modelo simples para estimar $\mathrm{R}_{\mathrm{s}}$ a partir da temperatura; referido modelo serviu de base para novos modelos já propostos ao longo do tempo.

Embora sejam modelos simples, derivados empiricamente, os modelos baseados na temperatura são fundamentados no conceito de que parte do saldo de radiação será utilizada na forma de calor sensível. O aumento de calor sensível durante o dia irá elevar a temperatura do ar acima daquela das condições noturnas e, assim, quanto maior a radiação maior também a temperatura do ar e, consequentemente, maior será a diferença entre a temperatura máxima e mínima do dia (Bristow \& Campbell, 1984).

Este método, entretanto, assume que a radiação solar é o principal fator meteorológico a condicionar a amplitude térmica, mesmo que outros fatores, como velocidade do vento, umidade do ar, disponibilidade hídrica no solo, altitude e precipitação, contribuam nos valores extremos da temperatura do ar, especialmente na escala diária (Allen, 1997). Desta forma, tornase urgente avaliar a sustentabilidade desses modelos no clima local, antes de serem usados como técnica operacional (Liu et al., 2009). Assim, ambos os modelos têm sido testados para diferentes regiões (Donatelli \& Campbell, 1998; Donatelli et al., 2003; Ball et al., 2004; Liu et al., 2009; Borges et al., 2010).

O objetivo deste estudo foi: a) calibrar os parâmetros de nove modelos empíricos de estimativa de $\mathrm{R}_{\mathrm{s}}$, a partir da temperatura, para as regiões Metropolitana, Vale do Rio Doce e Zona da Mata em Minas Gerais e b) avaliar os respectivos desempenhos.

\section{Material E MÉTODOS}

\section{Local e dados climáticos}

Utilizaram-se, para a realização deste estudo, os dados da temperatura do ar e a radiação solar dos anos de 2008 a 2010, obtidos por estações meteorológicas automáticas pertencentes ao Instituto Nacional de Meteorologia (INMET) disponíveis no endereço eletrônico da própria instituição (http:/ /www.inmet.gov.br). As regiões onde foram submetidos os estudos de calibração e performances dos modelos, foram a Metropolitana, Vale do Rio Doce e Zona da Mata, do Estado de Minas Gerais, que englobam as estações localizadas nos Municípios ilustrados na Figura 1.

A Tabela 1 apresenta a posição geográfica, o período de avaliação e a porcentagem de omissões das estações estudadas localizadas nos respectivos municípios mineiros. Os dados inicialmente reportados em intervalos horários foram

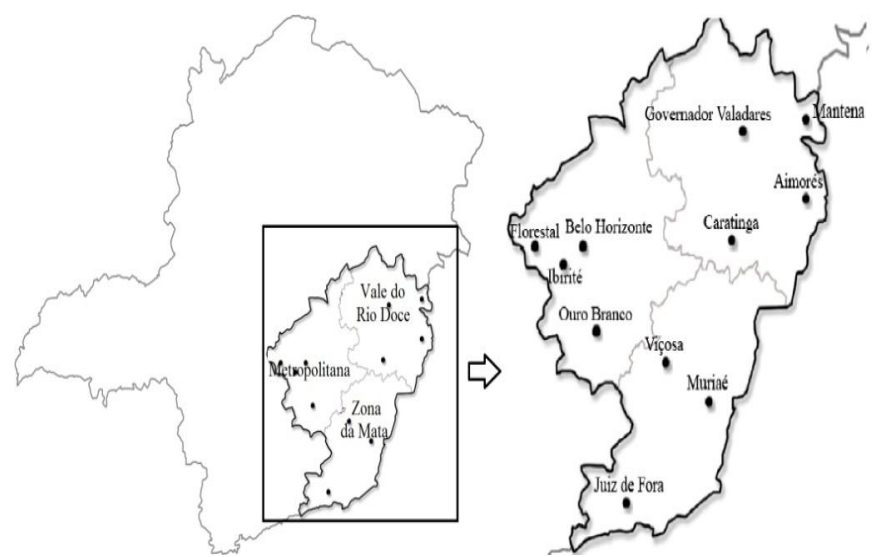

Figura 1. Localização das cidades utilizadas nas regiões Metropolitana, Vale do Rio Doce e Zona da Mata do Estado de Minas Gerais, Brasil 
Tabela 1. Localização geográfica, período e porcentagem de omissões das estações meteorológicas do Instituto $\mathrm{N}$ acional de M eteorologia (IN MET) utilizadas no presente estudo

\begin{tabular}{lrrrrc}
\hline \multicolumn{1}{c}{ Município } & Latitude & Longitude & Altitude & Período & Omissões (\%) \\
Aimorés & $-19,50^{\circ}$ & $-41,06^{\circ}$ & $193 \mathrm{~m}$ & $2008 / 09$ & 1,78 \\
B.Horizonte & $-19,88^{\circ}$ & $-43,97^{\circ}$ & $869 \mathrm{~m}$ & $2008 / 09$ & 1,78 \\
Caratinga & $-19,74^{\circ}$ & $-42,14^{\circ}$ & $615 \mathrm{~m}$ & $2008 / 09$ & 0,82 \\
Florestal & $-19,88^{\circ}$ & $-44,42^{\circ}$ & $742 \mathrm{~m}$ & $2009 / 10$ & 0,82 \\
Gov.Valadares & $-18,79^{\circ}$ & $-41,99^{\circ}$ & $263 \mathrm{~m}$ & $2009 / 10$ & 2,88 \\
lbirité & $-20,03^{\circ}$ & $-44,01^{\circ}$ & $816 \mathrm{~m}$ & $2009 / 10$ & 1.64 \\
Juiz de Fora & $-21,77^{\circ}$ & $-43,36^{\circ}$ & $950 \mathrm{~m}$ & $2008 / 09$ & 0,96 \\
Mantena & $-18,78^{\circ}$ & $-40,99^{\circ}$ & $270 \mathrm{~m}$ & $2008 / 09$ & 0,68 \\
Muriaé & $-21,10^{\circ}$ & $-42,38^{\circ}$ & $270 \mathrm{~m}$ & $2009 / 10$ & 1,78 \\
O. Branco & $-20,56^{\circ}$ & $-43,76^{\circ}$ & $1052 \mathrm{~m}$ & $2008 / 10$ & 0,55 \\
Viçosa & $-20,77^{\circ}$ & $-42,87^{\circ}$ & $712 \mathrm{~m}$ & $2008 / 09$ & 1,92 \\
\hline
\end{tabular}

contabilizados para valores diários, obtendo-se valores máximos (Tmáx) e mínimos (Tmín) de temperatura e total diário de radiação solar incidente $\left(\mathrm{R}_{\mathrm{s}}\right)$; em seguida, foram submetidos a uma triagem para verificar sua integridade e coerência, para o que se utilizou o critério de eliminação feito por Liu et al. (2009): a) dados ausentes para qualquer um dos elementos Tmáx, Tmín ou $R_{s}$; b) Tmáx $\leq$ Tmín; c) $R_{s} / R_{a}>1$. As porcentagens de omissões apresentadas na Tabela 1 foram calculadas dividindose os dados diários perdidos pelo total de dias do período.

\section{Descrição dos modelos}

A Tabela 2 apresenta as equações empíricas utilizadas: Hargreaves (1981), Bristow \& Campbell (1984), De Jong \& Stewart (1993), Donatelli \& Campbell (1998), Hunt et al. (1998), Annandale et al. (2002) e Chen et al. (2004).

O modelo de Hargreaves é amplamente utilizado em razão da sua simplicidade sendo, inclusive, recomendado no boletim da FAO-56 para ser usado quando os dados de radiação estão ausentes ou de qualidade duvidosa (Allen et al., 1998). Neste modelo, $\mathrm{R}_{\mathrm{s}}$ relaciona-se de maneira linear com a raiz quadrada da diferença $\left(\Delta T_{1}\right)$ entre a temperatura máxima (Tmáx) e mínima do ar (Tmín) de vez que o modelo referido apresenta apenas um coeficiente (a) (Modelo Ha); apesar disto, Chen et al. (2004) e Hunt et al. (1998) o apresentaram com dois coeficientes (a,b) (Modelos Ha-1 e Ha-2). A partir do modelo inicial de Hargreaves, novas modificações foram sendo propostas: Chen et al. (2004) propuseram relacionar $\mathrm{R}_{\mathrm{s}}$ de maneira logarítmica com a raiz quadrada da diferença $\left(\Delta \mathrm{T}_{1}\right)$ (Modelo $\left.\mathrm{Ch}\right)$; já Annandale et al. (2002) introduziram uma nova variável para correção da altitude (Modelo An); por sua vez, De Jong \& Stewart (1993) e Hunt et al. (1998) introduziram uma correção para o efeito da precipitação pluvial (J-S, Hu).

Bristow \& Campbell (1984) propuseram uma equação na qual a temperatura do ar se situou como principal variável de entrada; no entanto, $R_{s}$ é função exponencial de "T (Modelo BC). Embora os coeficientes sejam empíricos, o coeficiente a representa a máxima transmissividade esperada para um dia de céu claro, o qual depende da altitude e da poluição atmosférica local enquanto os coeficientes b e c controlam a taxa na qual a varia com a amplitude térmica. Valores frequentes encontrados para esses coeficientes são: 0,7 para a, a faixa de 0,004 a 0,010 para b e 2,4 para c (Meza \& Varas, 2000). Neste modelo a amplitude térmica diária $\left(\Delta \mathrm{T}_{2}\right)$ é obtida com base na diferença entre a temperatura máxima e o valor médio de duas temperaturas mínimas em dias subsequentes, com o objetivo de reduzir o efeito em larga escala da entrada advectiva de massas de ar quente ou fria, no local. Posteriormente, Donatelli \& Campbell (1998) propuseram uma correção para reduzir o efeito da sazonalidade em $\mathrm{R}_{\mathrm{s}}$ (Modelo $\mathrm{D}-\mathrm{C}$ ) e parametrizaram $\Delta \mathrm{T}_{2}$ pelo $\Delta \mathrm{T}$ médio do mês $\left(\Delta \mathrm{T}_{\mathrm{m}}\right)$.

\section{Coeficientes de calibração e avaliação de desempenho}

No período avaliado, um ano foi utilizado para calibrar os coeficientes e o outro para avaliar o desempenho dos modelos. Os anos foram escolhidos de acordo com as menores omissões. Obtiveram-se os coeficientes dos modelos a partir do método dos mínimos quadrados da diferença entre os valores observados e os estimados.

Tabela 2. Resumo dos modelos de estimativas da radiação estudados

\begin{tabular}{|c|c|c|c|}
\hline Modelo & Equação* & Coeficientes & Fonte \\
\hline An & $\mathrm{R}_{\mathrm{s}}=\mathrm{a} \cdot\left(1+2,7 \times 10^{-5} \cdot \mathrm{alt}\right) \cdot \sqrt{\Delta \mathrm{T}_{1}} \cdot \mathrm{R}_{\mathrm{a}}$ & a & Annandale et al. (2002) \\
\hline$B-C$ & $\mathrm{R}_{\mathrm{s}}=\mathrm{a} \cdot\left[1-\exp \left(-\mathrm{b} \cdot \Delta \mathrm{T}_{2}^{\mathrm{c}}\right)\right] \cdot \mathrm{R}_{\mathrm{a}}$ & $a, b, c$ & Bristow \& Campbell (1984) \\
\hline $\mathrm{Ch}$ & $\mathrm{R}_{\mathrm{s}}=\left(\mathrm{a} \cdot \ln \Delta \mathrm{T}_{1}+\mathrm{b}\right) \cdot \mathrm{R}_{\mathrm{a}}$ & $a, b$ & Chen et al. (2004) \\
\hline$D-C$ & $\mathrm{R}_{\mathrm{s}}=\mathrm{a} \cdot\left[1-\exp \left(-\mathrm{b} \frac{\Delta \mathrm{T}_{2}^{\mathrm{c}}}{\Delta \mathrm{T}_{\mathrm{m}}}\right)\right] \cdot \mathrm{R}_{\mathrm{a}}$ & $a, b, c$ & Donatelli \& Campbell (1998) \\
\hline $\mathrm{Ha}$ & $\mathrm{R}_{\mathrm{s}}=\mathrm{a} \cdot \sqrt{\Delta \mathrm{T}_{1}} \cdot \mathrm{R}_{\mathrm{a}}$ & a & Hargreaves (1981) \\
\hline $\mathrm{Ha}-1$ & $\mathrm{R}_{\mathrm{s}}=\left(\mathrm{a} \cdot \sqrt{\Delta \mathrm{T}_{1}}+\mathrm{b}\right) \cdot \mathrm{R}_{\mathrm{a}}$ & $a, b$ & Chen et al. (2004) \\
\hline $\mathrm{Ha}-2$ & $\mathrm{R}_{\mathrm{s}}=\mathrm{a} \cdot \sqrt{\Delta \mathrm{T}_{1}} \cdot \mathrm{R}_{\mathrm{a}}+\mathrm{b}$ & $a, b$ & Hunt et al. (1998) \\
\hline $\mathrm{Hu}$ & $\mathrm{R}_{\mathrm{s}}=\mathrm{a} \cdot \sqrt{\Delta \mathrm{T}_{1}} \cdot \mathrm{R}_{\mathrm{a}}+\mathrm{bT}_{\max }+\mathrm{cP}+\mathrm{dP}^{2}+\mathrm{e}$ & $a, b, c, d, e$ & Hunt et al. (1998) \\
\hline$J-S$ & $\mathrm{R}_{\mathrm{s}}=\mathrm{a} \cdot \Delta \mathrm{T}_{1}^{\mathrm{b}}\left(1+\mathrm{cP}+\mathrm{dP}^{2}\right) \cdot \mathrm{R}_{\mathrm{a}}$ & $a, b, c, d$ & De J ong \& Stewart (1993) \\
\hline
\end{tabular}


Para avaliar o desempenho de cada modelo de maneira similar aos trabalhos de avaliação de modelos de estimativa de $\mathrm{R}_{\mathrm{s}}$ foram utilizados os seguintes índices estatísticos: a) o coeficiente de determinação $\left(\mathrm{R}^{2}\right)$ das regressões lineares entre os valores observados e os estimados (Eq.1), b) raiz quadrada do quadrado médio do erro (RQME) (Eq. 2) e o c) erro médio (EM) (Eq. 3).

$$
\begin{aligned}
\mathrm{R}^{2}=1-\left[\frac{\sum_{\mathrm{i}=1}^{\mathrm{n}}\left(\mathrm{E}_{\mathrm{i}}-\mathrm{O}_{\mathrm{i}}\right)^{2}}{\sum_{\mathrm{i}=1}^{\mathrm{n}}\left(\mathrm{O}_{\mathrm{i}}-\overline{\mathrm{O}}\right)^{2}}\right] \\
\mathrm{RQME}=\left[\frac{1}{\mathrm{n}} \sum_{\mathrm{i}=1}^{\mathrm{n}}\left(\mathrm{E}_{\mathrm{i}}-\mathrm{O}_{\mathrm{i}}\right)^{2}\right]^{0,5} \\
\mathrm{EM}=\frac{1}{\mathrm{n}} \sum_{\mathrm{i}=1}^{\mathrm{n}}\left(\mathrm{E}_{\mathrm{i}}-\mathrm{O}_{\mathrm{i}}\right)
\end{aligned}
$$

em que:

$$
\begin{aligned}
& E_{1} \text { - valor estimado } \\
& O_{i} \text { - valor observado } \\
& \bar{O} \text { - média dos valores observados } \\
& n \text { - número de observações }
\end{aligned}
$$

A RQME varia entre 0 a infinito sendo que, quanto menor, melhor a estimativa de $\mathrm{R}_{\mathrm{s}}$; entretanto, este índice não faz distinção quanto aos dados serem subestimados ou superestimados (Jacovides \& Kontoyiannis, 1995). Similar ao RQME, quanto menor o valor absoluto de EM melhor a estimativa porém valores positivos indicam a quantidade média de superestimativa dos modelos, e negativos, o contrário.

Para verificar se, realmente, os valores de $\mathrm{R}_{\mathrm{s}}$ estimados pelos modelos diferiram significativamente entre os valores de $R_{s}$ medidos, utilizou-se o teste-t derivado a partir do RQME e EM (Stone, 1993; Togrul \& Togrul, 2002) (Eq. 4).

$$
\mathrm{t}=\left[\frac{(\mathrm{n}-1) \mathrm{EM}^{2}}{\mathrm{RQME}^{2}-\mathrm{EM}^{2}}\right]^{0,5}
$$

Quanto menor o valor de t melhor o desempenho do modelo, uma vez que a média das diferenças entre $\mathrm{E}_{\mathrm{i}}$ e $\mathrm{O}_{\mathrm{i}}$ será pequena. $\mathrm{O}$ valor crítico de $\mathrm{t}$ pode ser obtido na tabela estatística $\left(\mathrm{t}_{\text {tab, }} \mathrm{t}\right.$ Student), com nível de significância $(\alpha)$ e (n - 1) graus de liberdade. Um modelo será considerado estatisticamente significativo (aceitando a hipótese de nulidade, $\mathrm{H}_{\mathrm{o}}: \mathrm{E}_{\mathrm{i}}=\mathrm{O}_{\mathrm{i}}$ ) no intervalo $(1-\alpha)$, se o valor calculado de $t$ for menor que o valor crítico. No presente estudo o nível de significância escolhido foi de $0,05 \mathrm{com} \mathrm{n}=3.944 \mathrm{e}_{\mathrm{tab}}=1,96$.

\section{RESULTADOS E DISCUSSÃO}

\section{Coeficientes de calibração}

Os coeficientes obtidos para cada modelo estão presentes na Tabela 3. Para o modelo de Ha, o coeficiente a variou de 0,141 a 0,176 com média de 0,161 . Esses valores foram, em média, $6,1 \%$ superiores aos obtidos por Liu et al. (2009) e 5,7\% aos obtidos por Almorox et al. (2011), embora próximos ao recomendado por Allen et al. (1998) para um local situado no interior do continente e não calibrado $(0,16)$, ressaltando a universalidade deste modelo mas, adicionando-se o segundo coeficiente ao modelo de Hargreaves (Ha-1 e Ha-2), nota-se que os coeficientes obtidos oscilaram, especialmente para o coeficiente b (Ha-2) e, de maneira geral, foram diferentes aos obtidos por Liu et al. (2009), indicando a necessidade de calibração local; além disso, para alguns dias especialmente com baixos valores de $\Delta \mathrm{T}$, os mencionados coeficientes resultaram em valores negativos para $\mathrm{R}_{\mathrm{s}}$, os quais foram descartados.

A correção para altitude presente no modelo An, praticamente não interferiu no valor do coeficiente a, o qual foi ligeiramente inferior ao $\mathrm{Ha}$, com valores oscilando de 0,141 a 0,175 e média de 0,159 , corroborando com Liu et al. (2009) e Almorox et al. (2011); já no modelo Ch os coeficientes variaram pouco entre as cidades, com os valores dentro da faixa esperada pelos autores, ou seja, a variando entre 0,16 a 0,42 e b variando entre $-0,45$ e 0,12 .

Os coeficientes obtidos no modelo B-C foram próximos à faixa sugerida por Meza \& Varas (2000), porém com valores um pouco superiores para o b e inferiores para o c. Com base no valor médio do coeficiente a verificou-se que o coeficiente de transmissividade da atmosfera para um dia de céu limpo, foi de 0,73 .

No modelo D-C a diferença entre o maior e menor valor dos coeficientes a, b e c, foi de 0,148, 0,196 e 1,149, respectivamente, mostrando que esses coeficientes oscilaram consideravelmente entre as cidades e o coeficiente $\mathrm{c}$ foi o mais afetado corroborando com os obtidos por Almorox et al. (2011). No entanto, Liu et al. (2009) também verificaram oscilação semelhante porém o coeficiente $b$ foi o mais variável.

Para o modelo J-S, os coeficientes a e b variaram de 0,053 a 0,103 e 0,692 a 1,028 , respectivamente; por sua vez, os coeficientes c e d, que calibram os dados de precipitação, apresentaram valores muito pequenos, sinalizando que a inclusão da precipitação exerceu pouca influência na estimativa da $\mathrm{R}_{\mathrm{s}}$ concordando com os obtidos por Liu et al. (2009).

Os coeficientes do modelo $\mathrm{Hu}$ variaram de forma considerável entre as cidades, sendo que o coeficiente $\mathrm{d}$ teve a maior variação. Liu et al. (2009) encontraram valores ainda mais discrepantes entre os locais avaliados por eles, em que o coeficiente $b$ foi o que apresentou a maior variação.

As diferenças consideráveis entre os maiores e menores valores de cada coeficiente para os respectivos modelos aqui avaliados, indicam que seu ajuste local pode ser determinante no seu desempenho, conforme relatam alguns autores sobre a necessidade de calibração local em alguns modelos (Hargreaves, 1981; Mesa \& Varas, 2000). 
Tabela 3. Coeficientes de calibração dos modelos avaliados

\begin{tabular}{|c|c|c|c|c|c|c|c|c|c|c|c|c|}
\hline \multirow{2}{*}{$\begin{array}{c}\text { Modelos } \\
\text { Coeficientes }\end{array}$} & \multirow{2}{*}{$\begin{array}{c}\text { An } \\
\text { a }\end{array}$} & \multicolumn{3}{|c|}{ B-C } & \multicolumn{2}{|c|}{ Ha-1 } & \multicolumn{2}{|c|}{$\mathrm{Ch}$} & \multicolumn{4}{|c|}{$\mathrm{J}-\mathrm{S}$} \\
\hline & & a & b & c & a & b & a & b & a & b & c & d \\
\hline Aimorés & 0,175 & 0,734 & 0,016 & 1,924 & 0,300 & $-0,399$ & 0,423 & $-0,412$ & 0,076 & 0,860 & $-0,013$ & $2,6 \mathrm{E}-4$ \\
\hline Belo Horizonte & 0,167 & 0,713 & 0,009 & 2,222 & 0,315 & $-0,458$ & 0,417 & $-0,413$ & 0,103 & 0,739 & $-0,011$ & $6,8 \mathrm{E}-5$ \\
\hline Caratinga & 0,157 & 0,762 & 0,022 & 1,702 & 0,255 & $-0,309$ & 0,384 & $-0,375$ & 0,096 & 0,719 & $-0,010$ & $1,3 \mathrm{E}-4$ \\
\hline Florestal & 0,161 & 0,787 & 0,017 & 1,741 & 0,253 & $-0,325$ & 0,384 & $-0,387$ & 0,100 & 0,692 & $-0,003$ & $2,4 \mathrm{E}-5$ \\
\hline Governador Valadares & 0,162 & 0,763 & 0,027 & 1,607 & 0,254 & $-0,304$ & 0,368 & $-0,337$ & 0,088 & 0,757 & $-0,007$ & $7,7 \mathrm{E}-5$ \\
\hline Ibirité & 0,170 & 0,658 & 0,010 & 2,312 & 0,320 & $-0,446$ & 0,403 & $-0,365$ & 0,088 & 0,830 & $-0,018$ & $2,1 \mathrm{E}-4$ \\
\hline J uiz de Fora & 0,156 & 0,843 & 0,024 & 1,643 & 0,305 & $-0,424$ & 0,366 & $-0,308$ & 0,053 & 1,028 & $-0,016$ & $2,2 \mathrm{E}-4$ \\
\hline Mantena & 0,159 & 0,734 & 0,016 & 1,857 & 0,284 & $-0,404$ & 0,424 & $-0,470$ & 0,075 & 0,826 & $-0,017$ & $3,6 \mathrm{E}-4$ \\
\hline Muriaé & 0,155 & 0,662 & 0,020 & 1,850 & 0,243 & $-0,285$ & 0,364 & $-0,343$ & 0,090 & 0,744 & $-0,010$ & $1,3 \mathrm{E}-4$ \\
\hline Ouro Branco & 0,146 & 0,684 & 0,025 & 1,695 & 0,253 & $-0,328$ & 0,347 & $-0,317$ & 0,076 & 0,805 & $-0,011$ & $1,1 \mathrm{E}-4$ \\
\hline Viçosa & 0,141 & 0,741 & 0,017 & 1,724 & 0,240 & $-0,316$ & 0,342 & $-0,332$ & 0,067 & 0,817 & $-0,003$ & $1,9 \mathrm{E}-6$ \\
\hline Média & 0,159 & 0,735 & 0,018 & 1,843 & 0,275 & $-0,363$ & 0,384 & $-0,369$ & 0,083 & 0,802 & $-0,011$ & $1,4 \mathrm{E}-4$ \\
\hline Modelos & \multicolumn{3}{|c|}{ D-C } & $\mathrm{Ha}$ & \multicolumn{2}{|c|}{$\mathrm{Ha}-2$} & \multicolumn{5}{|c|}{$\mathrm{Hu}$} & \\
\hline Coeficientes & a & b & c & a & a & b & a & b & c & $d$ & e & \\
\hline Aimorés & 0,717 & 0,059 & 2,441 & 0,176 & 0,218 & $-4,646$ & 0,139 & 0,802 & $-0,238$ & 0,004 & $-20,345$ & \\
\hline Belo Horizonte & 0,662 & 0,027 & 2,851 & 0,170 & 0,171 & $-0,102$ & 0,121 & 0,522 & $-0,209$ & 0,001 & $-7,934$ & \\
\hline Caratinga & 0,751 & 0,092 & 1,702 & 0,159 & 0,218 & $-6,701$ & 0,158 & 0,495 & $-0,243$ & 0,003 & $-13,238$ & \\
\hline Florestal & 0,780 & 0,108 & 2,028 & 0,165 & 0,217 & $-6,687$ & 0,181 & 0,245 & $-0,145$ & 0,001 & $-8,751$ & \\
\hline Governador Valadares & 0,738 & 0,223 & 1,758 & 0,163 & 0,212 & $-5,774$ & 0,162 & 0,439 & $-0,195$ & 0,002 & $-13,120$ & \\
\hline lbirité & 0,632 & 0,040 & 2,768 & 0,173 & 0,147 & 2,788 & 0,105 & 0,581 & $-0,380$ & 0,004 & $-6,388$ & \\
\hline J uiz de Fora & 0,686 & 0,110 & 2,093 & 0,160 & 0,173 & $-1,357$ & 0,081 & 0,930 & $-0,363$ & 0,005 & $-13,304$ & \\
\hline Mantena & 0,687 & 0,070 & 2,302 & 0,160 & 0,190 & $-3,369$ & 0,102 & 0,857 & $-0,328$ & 0,007 & $-18,933$ & \\
\hline Muriaé & 0,656 & 0,157 & 1,988 & 0,156 & 0,186 & $-3,467$ & 0,137 & 0,454 & $-0,215$ & 0,002 & $-10,865$ & \\
\hline Ouro Branco & 0,639 & 0,123 & 2,058 & 0,150 & 0,173 & $-2,587$ & 0,121 & 0,529 & $-0,233$ & 0,002 & $-9,511$ & \\
\hline Viçosa & 0,701 & 0,075 & 2,144 & 0,144 & 0,184 & $-4,627$ & 0,112 & 0,682 & $-0,160$ & 0,001 & $-13,802$ & \\
\hline Média & 0,695 & 0,099 & 2,194 & 0,161 & 0,190 & $-3,321$ & 0,129 & 0,594 & $-0,246$ & 0,003 & $-12,381$ & \\
\hline
\end{tabular}

* An - Annandale et al. (2002); B-C - Bristow \& Campbell (1984); Ch - Chen et al. (2004); D-C - Donatelli \& Campbell (1998); Ha - Hargreaves (1981); Ha-1 - Chen et al. (2004); Ha-2 - Hunt et al. (1998); Hu - Hunt et al. (1998); J-S - De J ong \& Stewart (1993)

\section{Desempenho dos modelos}

Os valores de EM e RQME dos modelos estão apresentados na Figura $2 \mathrm{~A}$ e B. As regressões entre os pares de dados de $\mathrm{R}_{\mathrm{s}}$ observadas e estimadas pelos modelos e os valores obtidos em cada modelo para $\mathrm{R}^{2} \mathrm{e}$ a dispersão dos pontos em torno da reta $1: 1$, estão presentes na Figura 3 .
Seja para $\mathrm{R}^{2}$ ou para RQME, os resultados estão próximos aos valores obtidos em trabalhos de avaliação de modelos de estimativa de $\mathrm{R}_{\mathrm{s}}$ p partir da temperatura. De maneira geral, os valores de RQME aqui obtidos foram inferiores aos determinados por Liu et al. (2009) para as condições climáticas da China e Fortin et al. (2008) para o Canadá, porém superiores aos encontrados por Almorox et al.
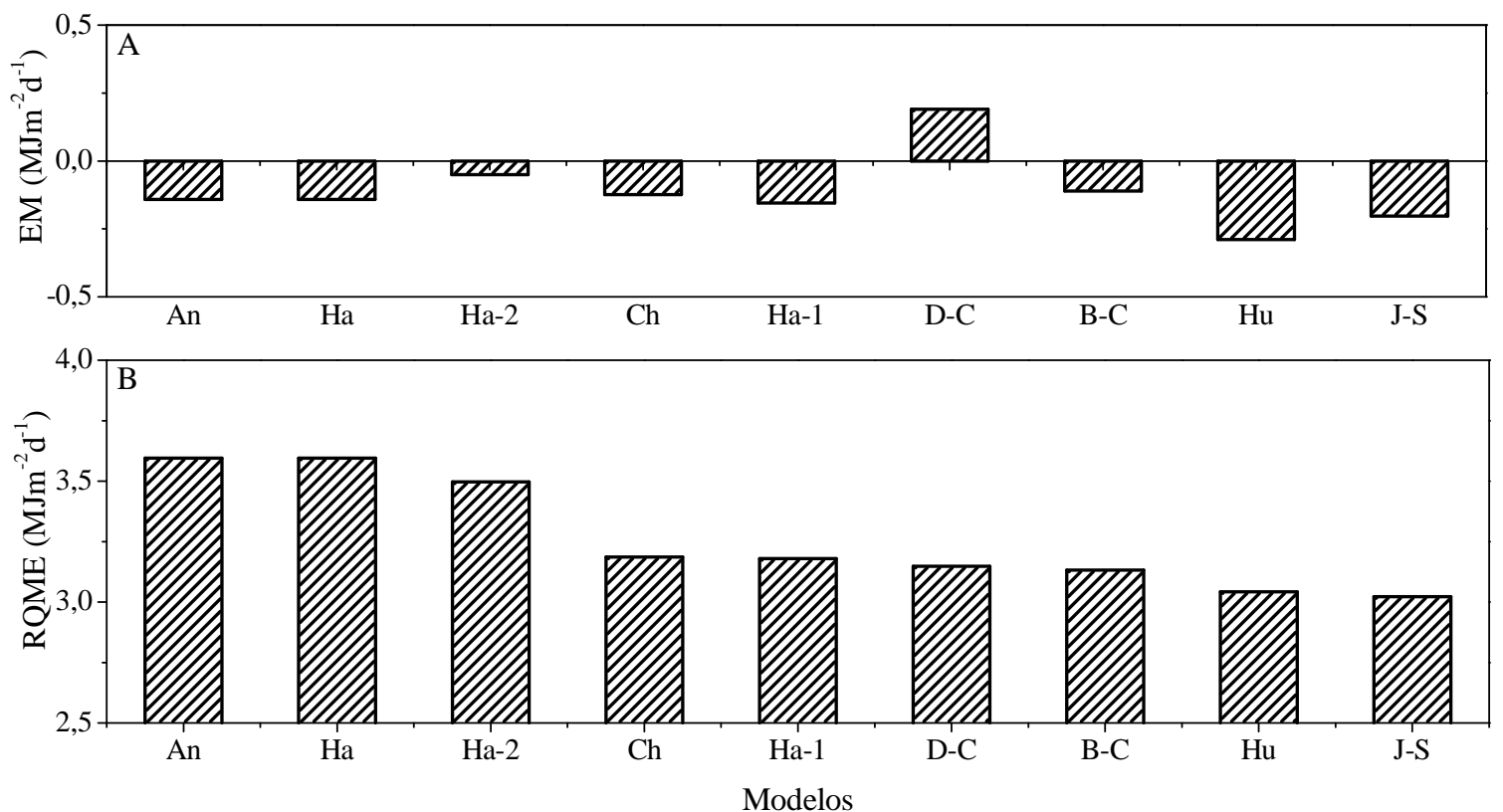

An - Annandale et al. (2002); B-C - Bristow \& Campbell (1984); Ch - Chen et al. (2004); D-C - Donatelli \& Campbell (1998); Ha - Hargreaves (1981); Ha-1 - Chen et al. (2004); Ha-2 - Hunt et al. (1998); Hu - Hunt et al. (1998); J-S - De Jong \& Stewart (1993)

Figura 2. Erro médio - EM (A) e raiz quadrada do quadrado médio do erro - RQ ME (B) das estimativa de $R_{s}$ feitas pelos model os 

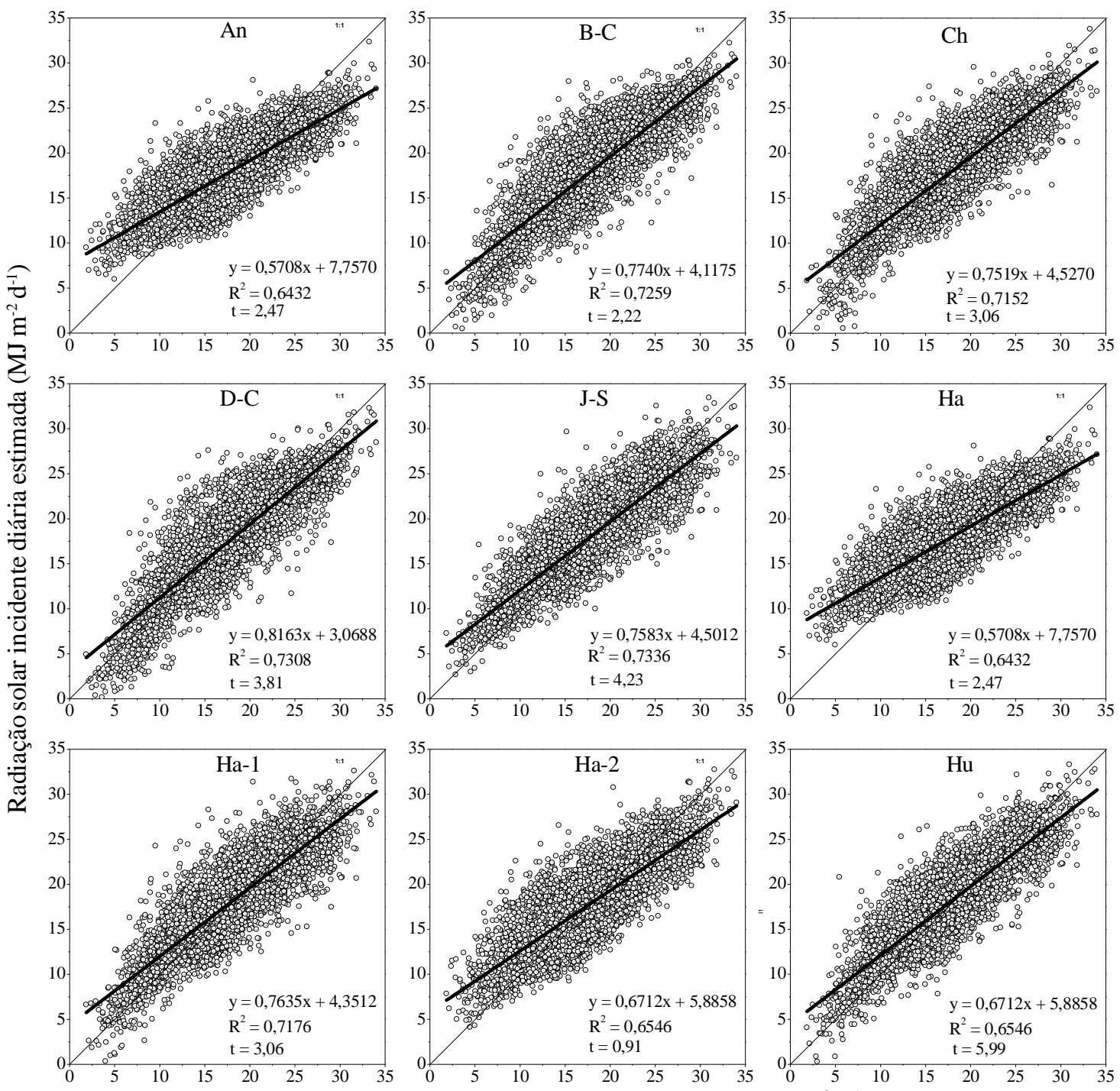

Obs.: $\mathrm{n}=3.944$ pontos

Radiação solar incidente diária observada $\left(\mathrm{MJ} \mathrm{m}^{-2} \mathrm{~d}^{-1}\right)$

Figura 3. Radiação solar incidente diária (Rs) observada e estimada pelos modelos

(2011) na Espanha. Borges et al. (2010) encontraram, em Cruz das Almas, BA, valores na faixa de 3,54 a $10,43 \mathrm{MJ} \mathrm{m}^{-2} \mathrm{~d}^{-1} \mathrm{e} 0,68$ a 0,71 para RQME e $\mathrm{R}^{2}$, respectivamente.

Comparativamente, é possível identificar dois grupos: o primeiro (Grupo I), de melhor desempenho, composto dos modelos de Hu, Ha-1, Ch, D-C, B-C e J-S que apresentaram RQME em torno de $3,1 \mathrm{MJ} \mathrm{m}^{-2} \mathrm{~d}^{-1}$ explicando $72 \%$ da variação de $\mathrm{R}_{\mathrm{s}}$ e um segundo (Grupo II), de pior desempenho, composto dos modelos restantes, Ha, An e Ha-2, apresentaram RQME em torno de 3,6 $\mathrm{MJ} \mathrm{m}^{-2} \mathrm{~d}^{-1}$ explicando $64 \%$ da variação de $\mathrm{R}_{\mathrm{s}}$. Assim, para esses índices os modelos modificados de Hargreaves (modelos An e Ha-2) e os modificados de Bristow \& Campbell (modelo D-C) não melhoraram o desempenho quando comparados com os modelos originais. Em geral, em modelagem quanto maior o número de variáveis uma equação possui melhores são as chances de o modelo conseguir explicar, de maneira mais clara, o fenômeno e, portanto, terá melhor ajuste (Liu \& Scott, 2001); no entanto, torna-se mais restritivo, tanto matematicamente como na disponibilidade dos dados; assim, neste trabalho a inclusão da precipitação e altitude não melhorou a sensibilidade às variações em $R_{s}$. Portanto, analisando os modelos do grupo I, tem-se que os modelos de Ha-1 e Ch, se tornam mais atrativos em razão da simplicidade dos modelos e pela facilidade na derivação dos coeficientes quando comparados com os demais.

Apesar disto, os resultados do EM foram diferentes daqueles para $\mathrm{R}^{2}$ e RQME. Na Figura 2A são apresentados os dados de EM. O menor desvio $\left(-0,05 \mathrm{MJ} \mathrm{m}^{-2} \mathrm{~d}^{-1}\right)$ ocorreu para o modelo de Ha-2 seguido dos modelos de B-C e Ch. O maior valor foi encontrado no modelo de $\mathrm{Hu}\left(-0,20 \mathrm{MJ} \mathrm{m}^{-2} \mathrm{~d}^{-1}\right)$. Com exceção do modelo de D-C, todos os modelos subestimaram, R. Notase então, observando a reta 1:1 (Figura 3), que a subestimativa recaiu especialmente sobre valores altos de $\mathrm{R}_{\mathrm{s}}$ mas se verificou, ainda, uma superestimava para valores baixos. Liu et al. (2009) também constataram tendência semelhante na China, e Borges et al. (2010) para o modelo de Hargreaves, em Cruz das Almas, em que a grande desvantagem na utilização do EM de forma isolada é a superestimava de uma observação individual, passível de ser anulada por uma subestimativa, em outra observação (Stone, 1993). 
Desta forma, ao trabalhar com índices estatísticos de maneira isolada, pode-se possibilitar que um modelo seja considerado insatisfatório, com base em determinado índice e satisfatório para outro, e vice-versa (Rivington et al., 2005; Abraha \& Savage, 2008). Para integrar os dois índices, alguns trabalhos de estimativa de radiação têm incorporado o teste t (Toðrul \& Toðrul, 2002; Almorox et al., 2008; Robba, 2009; Liu et al., 2009). Neste estudo e considerando o teste $t$ apenas para o modelo de Ha-2, as estimativas de $\mathrm{R}$ não diferiram, a nível de 0,05 de probabilidade, dos valores observados, uma vez que $\mathrm{t}=0,91<$ $\mathrm{t}_{0,05}=1,96$. Interessante observar que este modelo estava classificado no grupo II para $\mathrm{R}^{2}$ e RQME. Desta maneira, o teste $\mathrm{t}$ foi mais afetado pelo EM que pela RQME. Com base nos resultados obtidos neste trabalho, seria interessante que em estudos futuros este índice seja incluído para possibilitar sua validação ou não.

É importante salientar que as calibrações dos coeficientes foram feitas com dados de um ano e avaliado no ano posterior. É bem provável que se essa calibração tivesse sido feita em um período de tempo maior, o desempenho seria melhor, conforme verificaram Wu et al. (2007) ao comparar um período de cinco anos de calibração com um de doze anos, quando obtiveram melhor desempenho para o período de calibração maior.

\section{Conclusões}

1. Na ausência de dados medidos de radiação solar incidente, estimativas podem ser feitas a partir dos dados da temperatura máxima e mínima do ar, usando-se modelos empíricos de estimativa.

2. A pequena diferença entre os modelos avaliados e a proximidade com resultados encontrados na literatura, indica que qualquer um desses modelos pode ser empregado porém, dado à simplicidade, ao desempenho e à significância, o modelo de Hargreaves, calibrado e com dois coeficientes, é mais atrativo para as regiões Metropolitana, Vale do Rio Doce e Zona da Mata em Minas Gerais.

\section{LITERATURA CITADA}

Abraha, M. G., Savage, M. J. Comparison of estimates of daily solar radiation from air temperature range for application in crop simulations. Agricultural and Forest Meteorology, v.148, p.401-416, 2008.

Allen, R.G. Self-calibration method for estimating solar radiation from air temperature. Journal of Hydrologic Engineering, v.2, p.56-67, 1997.

Allen, R. G; Pereira, L. S.; Raes, D.; Smith, M. Cropevapotranspiration: guidelines for computing crop water requirements. Rome: FAO, 1998. 300p. Irrigation and Drainage Paper, 56.

Almorox, J. B.; Benito, M; Hontoria, C. Estimación de la radiación solar global en Venezuela. Interciencia, v.33, p.280283, 2008.

Almorox, J.; Hontoria, C.; Benito, M. Models for obtaining daily global solar radiation with measured air temperature data in Madrid (Spain). Applied Energy, v.88, p.1703-1709, 2011.
Al-Salaymeh, A. Model for the prediction of global daily solar radiation on horizontal surfaces for Amman city. Emirates Journal of Engineering Research, v.11, p.49-56, 2006.

Annandale, J. G; Jovanic, N. Z.; Benade, N.; Allen, R. G. Software for missing data error analysis of Penman-Monteith reference evapotranspiration. Irrigation Science, v.21, p.5767, 2002.

Ball, R. A.; Purcell, L. C.; Carey, S. K. Evaluation of solar radiation prediction models in North America. Agronomy Journal, v.96, p.391-397, 2004.

Borges, V. P.; Oliveira, A. S. de; Coelho Filho, M. C., Silva, T. S. M. da; Pamponet, R. M. Avaliação de modelos de estimativa da radiação solar incidente em Cruz das Almas, Bahia. Revista Brasileira de Engenharia Agrícola e Ambiental, v.14, p.7480, 2010.

Bristow, K. L.; Campbell, G. S. On the relationship between incoming solar radiation and daily maximum and minimum temperature. Agricultural and Forest Meteorology, v.31, p.159-166, 1984.

Chen, R. S.; Ersi, K.; Yang, J. P.; Lu, S. H.; Zhao, W. Z. Validation of five global radiation models with measured daily data in China. Energy Conversion and Management, v.45, p.17591769, 2004.

De Jong, R.; Stewart, D. W. Estimating global solar radiation from common meteorological observations in western Canada. Canadian Journal of Plant Science, v.73, p.509-518, 1993.

Donatelli, M.; Bellocchi, G.; Fontana, F. RadEst3.00: Software to estimate daily radiation data from commonly available meteorological variables. European Journal of Agronomy, v.18, p.363-367, 2003

Donatelli, M.; Campbell, G. S. A simple model to estimate global solar radiation. In: Congress of the European Society for Agronomy, 5, 1998. Procedings...Nitra: European Society for Agronomy, 1998. p.133-134.

Dornelas, K. D. S; Silva, C. L. da; Silva, C. A. da. Coeficientes médios da equação de Angström-Prescott, radiação solar e evapotranspiração de referência em Brasília. Pesquisa Agropecuária Brasileira, v.41, p.1213-1219, 2006

Fortin, J. G.; Anctil, León-Etiénne, P.; Bolinder, M. A. Comparison of empirical daily surface incoming solar radiation models. Agricultural and forest meteorology, v.14, p.1331-1340, 2008.

Hargreaves, G. H. Responding to tropical climates. In: The 198081 Food and climate review, the food and climate forum. Boulder: Aspen Institute for Humanistic Studies, 1981. p.2932.

Hunt, L. A.; Kuchar, L.; Swanton, C. J. Estimation of solar radiation for use in crop modeling. Agricultural and Forest Meteorology, v.91, p.293-300, 1998.

Jacovides, C. P.; Kontoyiannis, H. Statistical procedures for the evaluation of evapotranspiration computing models. Agricultural Water Management, v.27, p.365-371, 1995.

Kaplanis, S; Kaplani, E. A model to predict expected mean and stochastic hourly global solar radiation $\mathrm{I}(\mathrm{h} ; \mathrm{nj})$ values. Renewable Energy, v.32, p.1414-1425, 2007. 
Li, Huashan; Ma, W.; Lian, Y.; Wang, X. Estimating daily global solar radiation by day of year in China.Applied Energy, v.87, p.3011-3017, 2010.

Liu, D. L.; Scott, B. J. Estimation of solar radiation in Australia from rainfall and temperature observations. Agricultural and Forest Meteorology, v.106, p.41-59, 2001.

Liu, X.; Mei, X.; Li, Y.; Wang, Q.; Jensen, R. J.; Zhang, Y.; Porter, J. R. Evaluation of temperature-based global solar radiation models in China. Agricultural and Forest Meteorology, v.149, p.1433-1446, 2009.

Menges, H. O.; Ertekin, C.; Sonmete, M. H. Evaluation of global solar radiation models for Konya, Turkey. Energy Conversion and Management, v.47, p.3149-3173, 2006.

Meza, F.; Varas, E. Estimation of mean monthly solar global radiation as a function of temperature. Agricultural and Forest Meteorology, v.100, p.231-241, 2000.

Prieto, J. I.; Martínez-García, J. C.; García, D. Correlation between global solar irradiation and air temperature in Asturias, Spain. Solar Energy, v.83, p.1076-1085, 2009.
Rivington, M.; Bellochi, G.; Matthews, K. B.; Buchan, K. Evaluation of three model estimations of solar radiation at 24 UK stations. Agricultural and Forest Meteorology, v.132, p.228-243, 2005.

Robaa, S. M. Validation of the existing models for estimating global solar radiation over Egypt. Energy Conversion and Management, v.50, p.184-193, 2009.

Stone, R. J. Improved statistical procedure for the evaluation of solar radiation estimation models. Solar energy, v.51, p.289291, 1993.

Trnka, M.;Eitzinger, J.;Kapler, P.;Dubrovshý, M.;Semerádová, D.;Zalud, Z.; Formayer, H. Effect of estimated daily global solar radiation data on the results of crop growth models. Sensor, v.7, p.2330-2362, 2007.

TogrulI,T.; Togrul, H. Global solar radiation over Turkey: comparison of predicted and measured data. Renewable Energy, v.25, p.55-67, 2002.

Wu, G.; Liu, Y.; Wang, T. Methods and strategy for modeling daily global solar radiation with measured meteorological data - A case study in Nanchang station, China. Energy Conversion and Management, v.48, p.2447-2452, 2007. 